\title{
Comparing Psychological, Social, and Sexuality-Related Problems Reported by Bisexual and Gay Men: A Canadian Internet-Based Study
}

Kim Engler, Jean Dumas, Martin Blais, Joseph J. Lévy, Christine Thoër Université du Québec à Montréal

Bill Ryan
McGill University

Alain Léobon

Centre National de la Recherche Scientifique (France)

Barry Adam
University of Windsor

Kristopher Wells

University of Alberta

Blye Frank

Dalhousie University

Kim Engler, PhD, Montreal Chest Institute and Université du Québec à Montréal; Jean Dumas, PhD, Réseau de Recherche en Santé des Populations du Québec and Université du Québec à Montréal; Martin Blais, PhD, Department of Sexology, Université du Québec à Montréal; Joseph J. Lévy, PhD, Department of Sexology, Université du Québec à Montréal; Christine Thoër, PhD, Department of Social and Public Communication, Université du Québec à Montréal; Bill Ryan, MEd, MSW, School of Social Work, McGill University; Alain Léobon, PhD, Centre National de la Recherche Scientifique, Laboratoire CARTA, Angers, France; Barry Adam, $\mathrm{PhD}$, Department of Sociology and Anthropology, University of Windsor; Kristopher Wells, PhD, Institute of Sexual Minority Studies and Services, University of Alberta; Blye Frank, PhD, Division of Medical Education, Faculty of Medicine, Dalhousie University

This research was made possible with funding from the Canadian Institutes of Health Research (CIHR 2009-03-MOP-201493) and the Fonds Québécois de Recherche sur la Société et la Culture awarded to the SVR research team (www.svr.uqam.ca) of which Martin Blais, Joseph J. Lévy, Christine Thoër, Line Chamberland, Bill Ryan, Barry Adam, Kristopher Wells, and Blye Frank are co-investigators or affiliate researchers. The authors wish to thank the anonymous reviewers for their insightful comments.

Correspondence for this article should be directed to Dr. Kim Engler. Email: kimcengler@gmail.com 


\begin{abstract}
Greater clarification is needed about the differences between gay and bisexual men's health issues. With data from a pan-Canadian online survey, bisexual $(n=564)$ and gay men $(n=1,109)$ were compared on 32 different psychological, social, and sexual self-perceived problems that they might have encountered over the past 12 months. Clear patterns emerged. Relative to gay men, bisexual men had significantly higher adjusted odds of different sexual orientation or identity problems and certain health behaviour problems but significantly lower odds of several mental health and HIV/STI-related problems.
\end{abstract}

Keywords: homosexuality, bisexuality, gay men, health, well-being, Canada

The health, both physical and mental, of gays, lesbians, bisexuals, and other sexual minorities is being re-evaluated to update our understanding and means of addressing it. Added to the consequences of the HIV epidemic, which has disproportionately affected gay and bisexual men, a number of other health issues in these populations are recognized and documented in North American research. In the United States, health disparities between gay and bisexual men, on the one hand, and heterosexual men, on the other, are observed in areas such as drug use, suicidal attempts or ideation, and mental health, to the disadvantage of the former (Haas et al., 2011; King et al., 2008). Syndemic theory (Stall, Friedman, \& Catania, 2008) was developed precisely to emphasize the action of multiple epidemics, hypothesizing that they mutually interact, reinforce one another, and collectively diminish gay men's overall health profile. Especially intended to explain the health of American urban gay men within a life-course perspective, syndemic theory proposes that these epidemics result largely from socially produced harms associated with homophobia, cultural marginalization, and minority stress (Meyer, 2007; Stall et al., 2008).

Although in some respects Canada may offer more favourable conditions for sexual minorities than those of the United States (e.g., in health care systems, recognition of same-sex relationships, anti-discrimination policies by sexual orientation, the parenting rights of sexual minorities; Smith, 2005; Tjepkema, 2008), research with gay, bisexual, and lesbian populations in this country nevertheless finds that they face several health issues to a greater extent and that onset can begin from an early age. Within the school environment, for example, studies have found that sexual minority youth are more likely to experience a homophobic act (Chamberland, Émond, Julien, Otis, \& Ryan, 2008), verbal harassment, purposeful exclusion, physical assault (Saewyc et al., 2007), bullying, and sexual harassment (Williams, Connolly, Pepler, \& Craig, 2003). Bisexual boys surveyed in 2003 in British Columbia were also found to have significantly lower levels than both homosexual and heterosexual boys of factors considered protective of health: family connectedness, school appreciation, school connectedness, and religious or spiritual identification (Saewyc et al., 2009). Hence, relative to their heterosexual peers, the safety and well-being of sexual minority youth are disproportionately threatened in many respects, which can affect the social determinants of their health with potentially wide-ranging and long-term effects (Dysart-Gale, 2010), not least of which is vulnerability to HIV.

Epidemiologically, men who have sex with men (MSM) remain the group most affected by the HIV epidemic in Canada. According to 2008 estimates, the highest proportion of new HIV infections among all exposure categories considered occurred in MSM (44\%), representing from 1,000 to 1,900 seroconversions (Public Health Agency of Canada, 2010). In recent population-based analyses using Canadian Community 
Health Survey (CCHS) data, the health profile of gay and bisexual men was found to differ in many respects from that of the heterosexual male population. Both groups had significantly higher odds than heterosexual men of reporting a mood or anxiety disorder and lifetime suicidality (Brennan, Ross, Dobinson, Veldhuizen, $\&$ Steele, 2010). However, they had lower odds of being overweight and were similar to heterosexual men in odds of having a respiratory condition, hypertension, poor or fair subjective physical health, and poor or fair subjective mental health. In a study concerned with health care use drawing on the same survey, Tjepkema (2008) found gay men, but not bisexual men, were significantly disadvantaged relative to heterosexual men on a number of chronic conditions and in taking a disability day for a physical ailment in the past two weeks. Bisexual and gay men, as compared with heterosexual men, also had higher odds of using health professionals who offer emotional or psychological support (Tjepkema, 2008). However, in adjusted analyses (e.g., for socio-demographic variables), only bisexual men were more likely to have had an unmet health care need in the past 12 months.

As the findings of a qualitative study conducted in Ontario suggest, differences between gay and bisexual men may be partially explained by the unique stressors bisexuals face in the social, interpersonal, and intrapersonal domains (Ross, Dobinson \& Eady, 2010), including societal and internalized "biphobia." Defined as "negativity, prejudice, or discrimination against bisexual people" (p. 496), it could add to the challenges bisexuals already experience due to homophobia and heterosexism.

These previous studies underscore some of the preoccupying dimensions of sexual minority health as well as the need to consider the particularities of each group. They do, however, have some methodological limitations that we hope to address with the present study. The study by Ross et al. (2010), which explores experiences of discrimination among bisexuals, uses an Ontarian sample. The applicability of its findings to a wider population needs verification. In addition, the CCHS research may under-represent some groups of gay and bisexual men (e.g., it relies on respondents' self-identifying as gay or bisexual) and employs data collected several years ago (2003-2005).

Based a sub-sample of MSM from across Canada, recruited recently via an online survey designed to reach members of all lesbian, bisexual, gay, and transsexual populations, the present study offers an analysis of differences between gay and bisexual men on a range of self-identified problems in the social, sexual, and psychological spheres. Perceived problems, as indicators of need, could help inform more holistic efforts to improve the health of gay and bisexual men that are sensitive to their specificities. Taking a wide variety of health issues into account will contribute to developing a more comprehensive understanding of gay and bisexual men's health and its associated determinants, as demanded by community leaders, researchers, and decision-makers in matters of gay health (CATIE, 2010; Dumas, Mensah, \& Rousseau, 2008; Pink Triangle Services, 2001; Ryan \& Chervin, 2000), in order to develop new programs of prevention and health promotion, perhaps especially for use online.

\section{METHODOLOGY}

\section{Procedures and Participants}

From July to December 2010, a survey assessing health-related uses of the Internet was available online in both of Canada's official languages for completion by lesbian, gay, bisexual, two-spirit, and transgender 
Canadians. It inquired about basic information (e.g., sociodemographic data, sexual orientation), general uses of the Internet, state of health and well-being, including the presence of a wide range of problems in different life spheres, health-related uses of the Internet and online services, including their perceived impacts and appreciation, and issues of health management. Here, however, we focus on reported problems.

Multiple strategies of recruitment were used, including emails to 118 lesbian, gay, bisexual, and transgender organizations (LGBT) and $121 \mathrm{HIV} /$ AIDS organizations across the country (with follow-up) to seek their help in the recruitment effort, a two-month online banner publicizing the study in Xtra!, a Canadian gay and lesbian news website, as well as an advertisement in the bulletin emailed to its readership, personal emails sent to over 11,000 profiles on the meeting site QCboy.ca, over 45,000 emails sent to members of Squirt.org, and emails sent to 82 Yahoo LGBT groups based in Canada. Hardcopy and electronic publicity material was designed and distributed for recruitment purposes. Participation in the survey was voluntary and without compensation. The project was approved by the Université du Québec à Montréal's institutional review board for ethical research with humans.

Of the 2,736 surveys begun online, some were excluded: participants not residing in Canada $(n=22)$, those declaring a heterosexual identity and being exclusively attracted to and sexually active with members of the opposite sex $(n=16)$, and surveys with too much missing or inconsistent data $(n=511)$. This left 2,187 that were $70 \%$ complete or more. Bisexual and gay men filled out 1,673 , or $76.5 \%$, of these surveys. For purposes of this study, men were considered bisexual if they indicated, "in general," having sex especially with women, as much with women as with men, or mostly with men $(n=564)$. Men were considered gay if they claimed to have sex exclusively with men $(n=1,109)$.

\section{Measures}

Sociodemographic variables used to describe the sample in this paper are age (continuous); language (categorical with two levels: based on whether a respondent filled out an English or French version of the survey); diagnosed HIV-positive status (categorical: yes or no); current relationship status (categorical with two levels: coupled with either a man or a woman, or not); immigration status (categorical with three levels: third-generation Canadian at least, second-generation immigrant, and first-generation immigrant); education (categorical with four levels: primary or high school, college or one to four years of university, a bachelor's degree, a graduate (master's or doctoral) degree); annual income before taxes (categorical with two levels: less than 30,000 CAD or 30,000 CAD or more); worker status (categorical with two levels: part or full-time paid work, or not); and religious practice (average frequency rating from 1, never or practically never, to 8 , more than once a week). All of these variables, regardless of statistical significance, were used as controls in the multivariate analyses. Most are proxies of social determinants of health (income, education, employment, and social integration or support, in the case of religious practice, relationship status and immigration status). Language is used as a control for potential cultural effects tied to linguistic preference across the country in relation to the two official tongues.

This paper concentrates on 32 problems (in a checklist-type format) that survey respondents could have encountered over the past 12 months in the following areas: sexual orientation and identity, discrimination 
and victimization, sexually transmitted infections, mental health/well-being, health behaviours, and relationships (see Tables 2 and 3 for a complete list and for, generally, how they were described to respondents). Respondents indicated whether they had faced these problems and if so whether they concerned themselves personally or a friend/family (i.e., "Indicate which of the following medical and psychosocial problems you or a friend/family member have experienced in the past 12 months"). Only respondents' personal experience of these problems is examined here.

\section{Analyses}

To compare bisexual and gay respondents, for univariate analyses with sociodemographic variables and problems, Pearson's Chi square was used with categorical variables and Student's $t$-test with continuous variables. Logistic regression analyses were employed to examine the effect of sexual orientation on each problem. A gay orientation served as the constant in these analyses. Both unadjusted odds ratios and odds ratios adjusted for the previously mentioned controls are provided. Age was controlled with both centered linear and squared values to adjust for non-linear age effects. Variance Inflation Factor values for independent variables varied from 1.05 to $1.40(M=1.16)$, indicating that multicollinearity is not an issue. All statistical analyses were performed with Stata 11 software (StataCorp, 2009).

\section{RESULTS}

\section{General Characteristics of the Groups}

There was no significant difference between the sexual orientation groups in their distribution across the various means of recruitment $\left(\chi^{2}[5]=8.04 ; p=0.154\right)$. Over half of the sample was recruited by email (53.2\%), 24.0\% through an advertisement placed on a website, $7.5 \%$ via a banner on a website, $2.4 \%$ by word of mouth, $2.3 \%$ through an ad placed in a LGBT (electronic) paper or magazine, and 10.5\% from another source of recruitment (e.g., a Yahoo group). Their distribution across the country, however, varied $\left(\chi^{2}[4]=20.44 ; p<0.0001\right)$. Most apparently, a greater proportion of bisexuals than gay men were located in the Prairies (28.3\% vs. $20.6 \%$ ). For the remaining regions, sample proportions are as follows: Quebec (bisexuals: $33.8 \%$ vs. gays: $39.0 \%$ ), Ontario (bisexuals: $20.0 \%$ vs. gays: $22.4 \%$ ), western Canada (bisexuals: 9.7\% vs. gays: $12.7 \%$ ), and Atlantic Canada (bisexuals: $8.1 \%$ vs. gays: $5.3 \%$ ).

Variables chosen as controls in the multivariate analyses and further characterizing the sample are presented in Table 1. Both sexual orientation groups proved similar in age, income, and worker status. However, fewer bisexual men filled out the survey in French, had earned a graduate degree, or were firstgeneration immigrants, while more were part of a couple. A smaller proportion of bisexual men had also been diagnosed as HIV positive.

There was no significant difference between the sexual orientation groups in the mean number of problems reported over the past 12 months. On average, the men had encountered over 6 (bisexual men: $M=6.5$; gay men: $M=6.6 ; t[1,750]=0.17, p=0.864)$. 
Table 1

Sample Characteristics and Controls Used in the Multiple Logistic Regression Analyses

\begin{tabular}{|c|c|c|c|c|c|}
\hline Variable & $\begin{array}{l}\text { Total } \\
(\%)\end{array}$ & $\begin{array}{l}\text { Gays } \\
(\%)\end{array}$ & $\begin{array}{c}\text { Bisexuals } \\
(\%)\end{array}$ & Statistic $(d f)$ & $p$ \\
\hline Language (French) & 37.1 & 39.2 & 33.0 & $\chi^{2}(1)=6.00$ & 0.014 \\
\hline Age (mean) & 44.5 & 44.3 & 44.9 & $t(1,1655)=0.79$ & 0.430 \\
\hline HIV status (positive) & 8.6 & 11.4 & 3.1 & $\chi^{2}(3)=30.83$ & 0.000 \\
\hline Income ( $30 \mathrm{~K}$ or more) & 69.2 & 61.0 & 71.7 & $\chi^{2}(1)=2.27$ & 0.130 \\
\hline Worker (full/part-time) & 64.9 & 65.6 & 63.7 & $\chi^{2}(1)=0.55$ & 0.458 \\
\hline Religious practice (frequency) & 5.8 & 6.0 & 5.6 & $t(1,1626)=4.52$ & $<0.001$ \\
\hline Relationship status (coupled) & 43.0 & 40.6 & 47.9 & $\chi^{2}(1)=7.99$ & 0.005 \\
\hline \multicolumn{6}{|l|}{ Immigration status } \\
\hline Canadian, 3rd generation or + & 74.8 & 72.9 & 78.5 & $\chi^{2}(2)=9.98$ & 0.007 \\
\hline Immigrant, 2nd generation & 11.7 & 11.7 & 11.8 & & \\
\hline Immigrant, 1st generation & 13.5 & 15.4 & 9.7 & & \\
\hline \multicolumn{6}{|l|}{ Education } \\
\hline High school or less & 21.1 & 20.0 & 23.3 & $\chi^{2}(3)=10.60$ & 0.014 \\
\hline College or some university & 39.0 & 37.5 & 42.0 & & \\
\hline Bachelor's degree & 23.7 & 24.6 & 21.8 & & \\
\hline Graduate degree & 16.2 & 17.9 & 12.8 & & \\
\hline
\end{tabular}

\section{Sexuality-Related Problems}

Crude estimates of the proportions of bisexual and gay men reporting problems of a more sexual nature are shown in Table 2. In this general area, the most prevalent in bisexual men were fearing others will discover their sexual orientation (43.0\%) and sexual risk practices and accepting their sexual orientation, reported by approximately a third each. In gay men, sexual health issues tied to sexually transmitted infection were most problematic; approximately $40.0 \%$ mentioned facing problems of HIV testing, other STI testing, and sexual risk practices.

Results of the logistic regression analyses on these types of self-perceived problems are also presented in Table 2. In general, they show not only multiple differences in odds between the sexual orientation groups but the control variables' lack of explanatory value for the pattern of significant findings, albeit with some notable exceptions. Net of controls, bisexual men were from about two to over three times more likely than gay men to report problems linked to their sexual orientation or gender identity, whether issues of acceptance with regard to these aspects, coming out, or fearing others will discover their sexual orientation. 


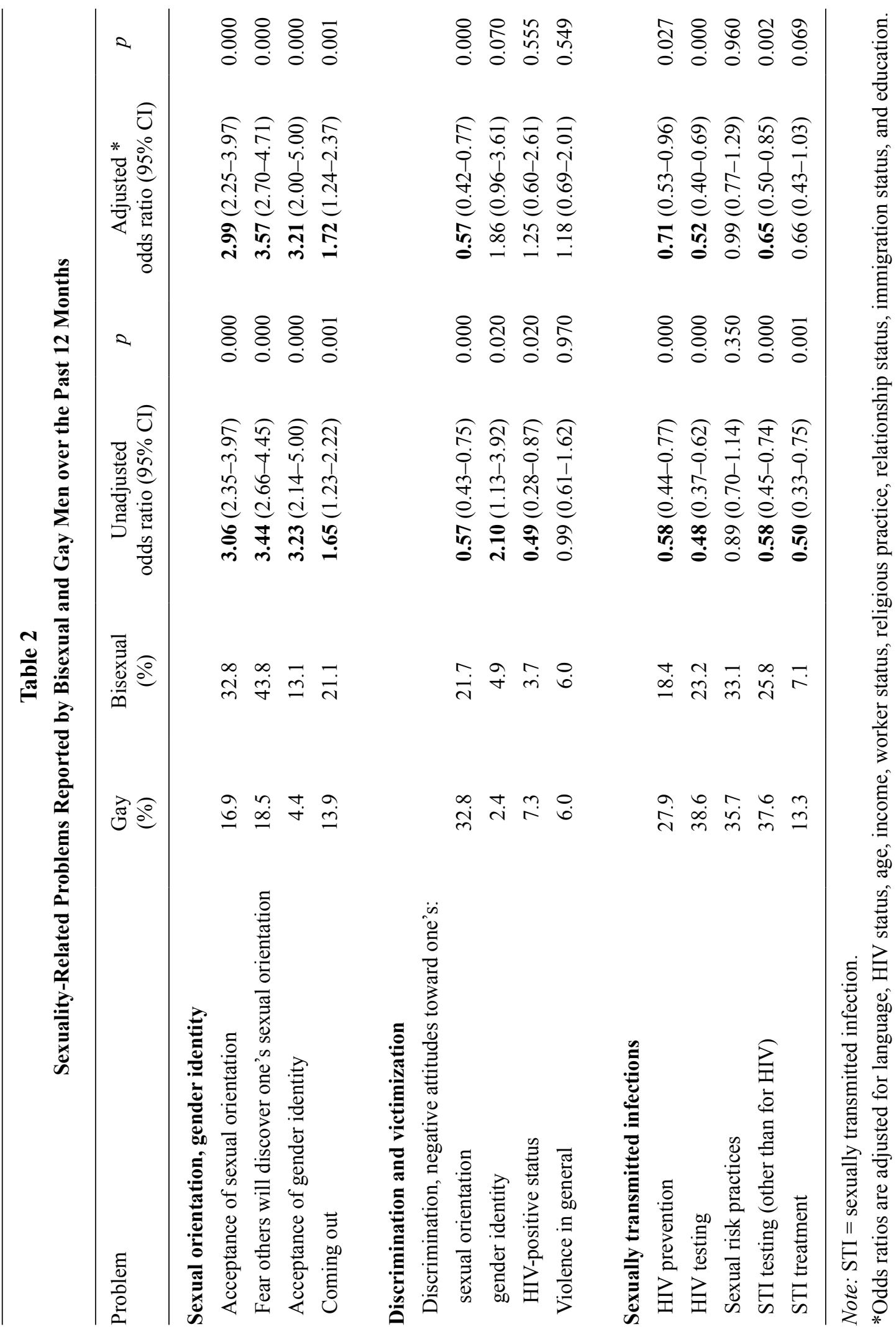


Dealing with discrimination or other people's negative attitudes toward their sexual orientation was, however, significantly less likely in this group. Lower adjusted odds of three problems tied to sexually transmitted infection (STI) were also observed. These concerned problems of HIV prevention, HIV testing, and other STI testing. Significantly lower unadjusted odds in bisexual men of reporting a problem with STI treatment and discrimination or others' negative attitudes toward one's HIV status attenuated to non-significance with the integration of controls (including HIV status). Bisexual men's significantly greater likelihood of having dealt with discrimination or negative attitudes with regard to their gender identity, when adjusted, also became non-significant. No significant difference was found between bisexual and gay men in odds of experiencing a problem with violence in general (an exception in this list of sexuality-related problems) or sexual risk practices.

\section{Psychological, Health-Behavioural, and Interpersonal Problems}

Table 3 also provides both descriptive statistics and the odds ratios generated by the regression analyses, in this case with regard to more psychological and interpersonal problems. Among these, for both sexual orientation groups, based on crude estimates, the mental health and well-being problems of body image and anxiety, in that order, were most prevalent. Approximately half of gay men reported dealing with each of them in the previous 12 months, while proportions were lower yet still quite high in bisexual men (body image: 45\%; anxiety: 38\%). Other problems affecting at least a third of gay men were depression, loneliness or isolation, finding friends, and relationship issues. Among bisexuals, a little less than a third had dealt with the problems of loneliness or isolation and finding a long-term partner.

Once again, the control variables proved to have little bearing on the overall pattern of significant odds found in the unadjusted regression analyses. Relative to gay men, bisexual men had significantly lower adjusted odds of problems with anxiety, body image, depression, and suicidal thoughts or behaviour in the past 12 months. Their likelihood of reporting a problem finding friends or a long-term partner was also lower. Greater odds of several types of health behaviour problems were, however, noted: compulsive sexuality, dependence on the Internet, and dependence on erotic images. While reported by a small proportion of men, the highest adjusted odds were found for violence in a relationship; these were over twice as high in bisexual men. With adjustment, initially lower odds of loneliness or isolation as well as smoking in bisexual men became non-significant. No significant difference in odds was seen for problems with an eating disorder, compulsive gambling, alcohol use, drug use, a break-up, family, or relationship issues.

\section{DISCUSSION}

With data from a pan-Canadian online survey, this study examined a broad spectrum of self-identified psychological, sexuality-related, and social problems in a sample of bisexual and gay men. Sexual orientation (bisexual versus gay), defined as a function of the sex of respondents' sexual partners, was associated with many perceived problems, independent of language, HIV status, age, income, education, religious practice, relationship status, worker status, and immigration status, as constructed in this study. Specifically, bisexual men were found to be significantly more concerned than gay men with sexual orientation or gender identity problems, issues of behavioural control, and relationship violence but significantly less concerned with sexual orientation discrimination, sexually transmitted infection, mental health, and relationship-finding issues. 
COMPARING PSYCHOLOGICAL, SOCIAL, AND SEXUALITY-RELATED PROBLEMS

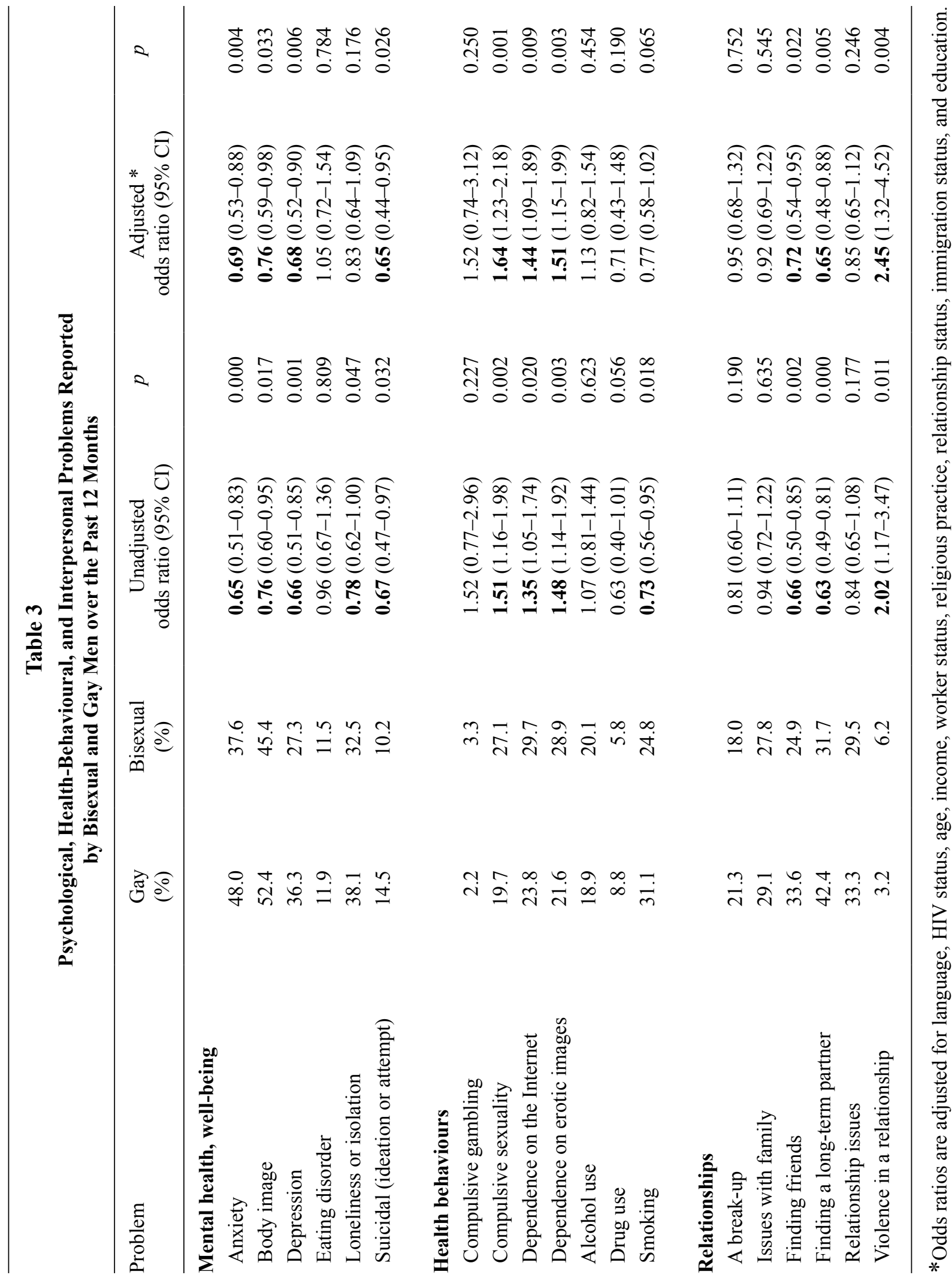


Greater odds of problems related to coming out, sexual orientation acceptance, sexual identity acceptance, and fearing others will discover one's sexual orientation were found in bisexual men. This is consistent with previous research indicating lower levels of sexual orientation disclosure in bisexual men relative to gay men (e.g., Doll et al. 1992), as well as higher internalized homophobia and less acceptance of same-sex behaviour (Stokes, Vanable, \& McKirnan, 1997). Bisexual men's greater perceived problems with gender identity could therefore signal worries about gender non-conformity (or femininity), which would threaten to publicly label them as a sexual minority.

Bisexual men's lower odds of problems with discrimination or the negative attitudes of others regarding their sexual orientation may be related to the previous findings. If they are less "out" and visible as a sexual minority, this may decrease their exposure to or perception of these types of events. Indeed, the Canadian General Social Survey of 2004 found that among those who experienced discrimination, 78\% of gays and lesbians versus $29 \%$ of bisexuals had attributed it to their sexual orientation (Beauchamp, 2008). This general pattern of results is observed in other relatively tolerant countries, where lower levels of negative reactions and openness as well as higher levels of internalized homonegativity are reported in bisexuals relative to gays (Kuyper \& Fokkema, 2011).

The odds of sexuality-related problems concerned with STI were significantly lower among bisexual men relative to gay men in several categories (i.e., HIV prevention, testing for HIV, testing for other STI). Many explanations are possible. Bisexual men may have fewer STI or engage in less testing. In Brennan et al. (2010), only $9.4 \%$ of Canadian bisexual men reported ever being diagnosed with an STI compared with $26.6 \%$ of gay men. Bisexual men may be less preoccupied with HIV risk or the possibility of acquiring a STI (Goldbaum, Perdue, \& Higgins, 1996) because they are less exposed to HIV/STI prevention programming due to a low level of contact with the gay milieu (e.g., McKirnan, Stokes, Doll, \& Burzette, 1995) or lesser proximity to an AIDS service organization (Leaver, Allman, Meyers, \& Veugelers, 2004). In our study, a greater proportion of bisexual men than gay men resided in more rural provinces, where resources could be more limited. Existing HIV/STI programs may also not adequately address heterosexual behaviour in bisexual men (Leaver et al., 2004). Finally, another explanation may be found in bisexual men's distance from the epidemic in the form of less contact with people known to have HIV/AIDS (Heckman et al., 1995; Myers, Allman, Jackson, \& Orr, 1995).

With respect to our findings on bisexual men's health-related behaviours, some authors have suggested that bisexual men, in an effort to conceal their sexual orientation, may seek out "anonymous, highly sexualized contexts" in which to live out their desires for same-sex sexuality (Stokes, Miller, \& Mundhenk, 1998). In Myers et al. (1995), Canadian bisexual men were more likely than gay men to be recruited in bathhouses. The Internet can provide such a space, allowing MSM a venue in which to explore their sexuality discreetly and with less fear of negative repercussions (Parsons, Severino, Grov, Bimbi, \& Morgenstern, 2007). The reinforcing features of the Internet (Parsons et al., 2007) may be conducive, under certain conditions, to dependence on this medium as well as to compulsive sexuality and dependence on erotic images, all problems that bisexual men in our sample had greater odds of reporting. It is worth highlighting, however, that at least a fifth of the gay men and bisexual men we recruited online reported a problem in each of these areas. As suggested by a recent review of Canadian organizational websites that provide information on the health of sexual minorities, very few (4-8\%) offer information on these topics (Levy, Dumas, Thöer, Ryan, 
\& Léobon, 2009). Although the Internet may not be the ideal space in which to do so, our findings suggest that there is a need to better address these issues.

In terms of mental health and well-being, relative to gay men, bisexual men were found to have significantly lower odds of problems with anxiety, depression, suicidal ideation or attempt, and body image. In Brennan et al. (2010), odds of a mood or anxiety disorder were also higher in gay men than in bisexual men (relative to heterosexual men). However, bisexual men had greater odds than gay men of lifetime suicidality (Brennan et al., 2010). The reason for these diverging results is not clear but may lie in differences between the studies in sample construction (e.g., sexual orientation measurement, recruitment).

Based on crude estimates, a problem with body image was reported by roughly half of both bisexual and gay men and was the most pervasive self-perceived problem among those considered. Observations of a higher prevalence of eating disorders in gay and bisexual men relative to heterosexual men (e.g., Feldman $\&$ Meyer, 2007) have generated a number of explanations for both ideals of thinness and muscularity centred on a range of psychological and socio-cultural factors, including the role of gay media and socialization processes, the need to attract male partners, and masculinity issues that touch all men to varying degrees (Kane, 2010). It is possible that bisexual men's lower odds of reporting a problem with body image reflects less connection with gay culture and concern with meeting its bodily ideals. As studies on this topic often combine gays and bisexuals in their samples (Kane, 2010), further investigation is needed into what drives body image issues in each group.

Relative to gay men, bisexual men in this study had lower odds of problems finding friends or a long-term relationship but greater odds of relationship violence. Internalized homophobia is linked with relationship problems in gays, lesbians, and bisexuals, including among the coupled (Frost \& Meyer, 2009). In Canada, while the difference was not significant, experiences of spousal abuse have been found in higher proportions among bisexuals (28\%) than in gays and lesbians (15\%; Beauchamp, 2008). Fears that their sexual orientation will be found out may keep bisexual men from seeking help, and the reality of limited resources for and social recognition of relationship violence among sexual minorities must not be overlooked (Kirkland, 2004). Lower odds of problems finding friends and a long-term partner may reflect gay men's greater reliance on gay milieus to meet many of their social needs (e.g., because of experiences of discrimination). If these are small or difficult to access, for instance, it may limit gay men's social networks (relative to that of bisexual men) or make establishing certain relationships more difficult.

Overall, both bisexual and gay men reported an average of over six health-related problems over the past 12 months. In addition, a problem with anxiety, depression, loneliness/isolation, sexual risk practices, or smoking was reported by at least a quarter of bisexual and gay men each. According to syndemic theory, as developed by Stall et al. (2008), cultural marginalization is sufficient to produce the pattern of multiple health issues observed in gay men. They identify two central processes through which syndemics are produced: harms linked to the early socialization of gay men (e.g., masculine socialization) and the stresses of migrating to "gay ghettos." The differentials we observed between gay and bisexual men in self-reported problems may indicate differences in these areas, for example, in responses to early socialization (e.g., more or less internalized homophobia), connection with sexual minority communities (e.g., affecting one's ability to pass as heterosexual, avoid homophobic attacks), and the general balance between stressors and strengths to which each group has access. These possibilities should be pursued in future research. 
This study has a number of limitations. While there is evidence that samples of men who have sex with men recruited online can approximate national probability samples of these men along certain sociodemographic and behavioural dimensions (Evans, Wiggins, Mercer, Bolding, \& Elford, 2007), our convenience Internet-based sample may nevertheless be unrepresentative of gay and bisexual Canadian men in the general population. Comparing our sample to that of gay and bisexual men recruited by the population-based Canadian Community Health Survey (CCHS) showed some differences on common variables. Relative to self-identified bisexual and gay men of the CCHS, as reported in Brennan et al. (2010), bisexual and gay men in our study seem somewhat older (bisexual men: $M=44.9$ vs. 39.3 ; gay men: $M=44.3$ vs. 39.9 ) and to contain fewer workers, especially among gays (bisexual men: $63.7 \%$ vs. $66.8 \%$; gay men: $65.6 \%$ vs. $72.7 \%$ ). One explanation may be our sample's wider age range (17-80 vs. 18-59 in Brennan et al., 2010). On education, a smaller proportion of bisexual men in our study (23.3\% vs. $32.0 \%)$ and a larger proportion of gay men (20.0\% vs. 14.6\%) had earned a high school degree or less (Tjepkema, 2008). The ratio of gay to bisexual men recruited, however, was similar between our sample (2 to 1) and that of the CCHS (2.2 to 1; Brennan et al., 2010; Tjepkema, 2008), but our online sample reflects the advantage of an anonymous, Internet-based recruitment for reaching large numbers of sexual minority men.

The measure of sexual orientation used in the present paper does not incorporate a defined timeframe for sexual behaviour nor does it take into account men's sexual orientation identity. Hence, the men composing the bisexual group may be quite heterogeneous, containing men transitioning to a gay identity, gay-identified men with previous heterosexual experience, and bisexual-identified men, for example (Stokes et al., 1998). Our findings on problems with one's sexual orientation do suggest, however, that the sample of bisexuals contains a good proportion of closeted men.

Our assessment of perceived health-related problems was modelled on a community-based approach, in a checklist format. As a result, the problems are succinctly defined and may mean different things to different respondents. They are not indicators of dysfunction or impairment, which may be best evaluated with multi-item measures. Future research could aim to examine many of the problem areas suggested by our checklist using diagnostic tools.

\section{CONCLUSION}

This Internet-based study of Canadian gay and bisexual men reinforces the pertinence of disaggregating groups according to sexual orientation for purposes of health-related analyses. Patterns of relative vulnerability were observed that indicate bisexual men are more likely to experience certain problems tied to sexual orientation and identity (e.g., coming out, fears others will discover one's sexual orientation) and health behaviour (e.g., dependence on the Internet) but are less likely to encounter some problems related to mental well-being (e.g., depression, anxiety, suicidality) and sexual health (e.g., HIV testing, STI testing). These findings could be used in an effort to develop programs aimed at addressing the broad health needs of gay and bisexual men that are sensitive to each group's specificities.

Consistent with syndemic theory, both bisexual and gay men reported an average of over six selfperceived problems over the past 12 months. The social factors (e.g., minority stressors) underlying them may vary between the groups, shaping the differences observed in their configuration. Future research could investigate this possibility. While this study focused on individually assessed health-related problems, it is 
recommended that further investigation be conducted on syndemic processes as well as on the assets and resiliencies in the gay and bisexual male populations (CATIE, 2010).

\section{RÉSUMÉ}

Les différences entre les hommes gais et les hommes bisexuels quant à leurs problématiques de santé méritent d'être mieux cernées. Dans le cadre d'une enquête en ligne pancanadienne, nous avons comparé des hommes bisexuels $(n=564)$ et gais $(n=1,109)$ à partir de 32 problèmes autoperçus d'ordre psychologique, social ou sexuel auxquels ils pouvaient avoir fait face au cours des 12 derniers mois. Les résultats indiquent que, par rapport aux hommes gais, les hommes bisexuels font face davantage aux problèmes touchant l'orientation ou l'identité sexuelle ou relatifs à certains comportements néfastes pour la santé, mais moins à plusieurs problèmes de santé mentale et à des problèmes liés aux ITS/VIH.

Mots clés : homosexualité, bisexualité, hommes gais, santé, bien-être, Canada

\section{REFERENCES}

Beauchamp, D.L. (2008). Sexual Orientation and Victimization. Canadian Centre for Justice Statistics, Statistics Canada.

Brennan, D.J., Ross, L.E., Dobinson, C., Veldhuizen, S., \& Steele, L.S. (2010). Men's sexual orientation and health in Canada. Canadian Journal of Public Health, 101(3), 255-258.

CATIE - Canadian AIDS Treatment Exchange. (2010). New Directions in Gay Men's Health and HIV Prevention in Canada: Pan-Canadian Deliberative Dialogue Report. Retrieved from http://library.catie.ca/PDF/ATI40000s/40224.pdf

Chamberland, L., Émond, G., Julien, D., Otis, J., \& Ryan, W. (2008). L'impact de l'homophobie et de la violence homophobe sur la persévérance et la réussite Scolaire. Rapport de Recherche. Fonds de Recherche sur la Société et la Culture. Retrieved from http://www.fqrsc.gouv.qc.ca/upload/editeur/RF-LineChamberland.pdf

Dumas, J., Mensah, M.N., \& Rousseau, R. (2008). Etes-vous satisfait? Peut-on faire mieux... autrement? Rapport de l'enquête auprès d'hommes ayant des relations sexuelles et affectives avec d'autres hommes sur l'approche globale de la santé et du mieux-être à Action Séro Zéro. Montreal, QC: Action Séro Zéro and the Département de Travail Social, Université du Québec à Montréal. Retrieved from http://www.rezosante.org/DATA/DOCUMENT/31_ fr $\sim$ V $\sim$ Rezo-documents-Etes-vous_satisfait_Peut-on_faire_mieux_autrement_2008_.pdf

Doll, L.S., Petersen, L.R., White, C.R., Johnson, E., Ward, J.W., \& The Blood Donor Study Group. (1992). Homosexually and non-homosexually identified men who have sex with men: A behavioral comparison. Journal of Sex Research, 29, 1-14.

Dysart-Gale, D. (2010). Social justice and social determinants of health: Lesbian, gay, bisexual, transgendered, intersexed, and queer youth in Canada. Journal of Child and Adolescent Psychiatric Nursing, 23(1), 23-28.

Evans, A.R., Wiggins, R.D., Mercer, C.H., Bolding, G.J., \& Elford, J. (2007). Men who have sex with men in Great Britain: Comparison of a self-selected internet sample with a national probability sample. Sexually Transmitted Infection, 83, 200-205.

Feldman, M.B., \& Meyer, I.H. (2007). Eating disorders in diverse lesbian, gay, and bisexual populations. International Journal of Eating Disorders, 40, 218-226.

Frost, D.M., \& Meyer, I.H. (2009). Internalized homophobia and relationship quality among lesbians, gay men, and bisexuals. Journal of Counseling Psychology, 56(1), 97-109.

Goldbaum, G., Perdue, T., \& Higgins, D. (1996). Non-gay-identifying men who have sex with men: Formative research reports from Seattle, Washington. Behavioral Science in HIV Prevention, III (suppl. 1), 36-40.

Haas, A.P., Eliason, M., Mays, V.M., Mathy, R.M., Cochran, S.D., D’Augelli, A.R., . . Clayton, P. J. (2011). Suicide and suicide risk in lesbian, gay, bisexual, and transgender populations: Review and recommendations. Journal of Homosexuality, 58, 10-51.

Heckman, T.G., Kelly, J.A., Sikkema, K.J., Roffman, R.R., Solomon, L.J., Winett, R.A., . . Desiderato, L.J. (1995). Differences in HIV risk characteristics between bisexual and exclusively gay men. AIDS Education and Prevention, 7, 504-512. 
Kane, G.D. (2010). Revisiting gay men's body image issues: Exposing the fault lines. Review of General Psychology, 14(4), 311-317.

King, M., Semlyen, J., Tai, S.S., Killaspy, H., Osborn, D., Popelyuk, D., \& Nazareth, I. (2008). A systematic review of mental disorder, suicide, and deliberate self harm in lesbian, gay, and bisexual people. BMC Psychiatry, 8(70).

Kirkland, K. (2004). Abuse in Gay Male Relationships: A Discussion Paper. Government of Canada: National Clearinghouse on Family Violence.

Kuyper, L., \& Fokkema, T. (2011). Minority stress and mental health among Dutch LGBs: Examination of differences between sex and sexual orientation. Journal of Counseling Psychology, 58(2), 222-233.

Leaver, C.A., Allman, D., Meyers, T., \& Veugelers, P.J. (2004). Effectiveness of HIV prevention in Ontario, Canada: A multilevel comparison of bisexual men. American Journal of Public Health, 94(7), 1181-1185.

Levy, J.J., Dumas, J., Thöer, C., Ryan, B., \& Léobon, A. (2009). Internet et santé des minorités sexuelles au Canada: Une étude exploratoire. Santé Publique, 53-64.

McKirnan, D.J., Stokes, J.P., Doll, L., \& Burzette, R.G. (1995). Bisexually active men: Social characteristics and sexual behavior. The Journal of Sex Research, 32, 65-76.

Meyer, I.H. (2007). Prejudice and discrimination as social stressors. In I.H. Meyer \& M.E. Northbridge (Eds.), The Health of Sexual Minorities: Public Health Perspectives on Lesbian, Gay, Bisexual and Transgender Populations (pp. 242-267). New York, NY: Springer.

Myers, T., Allman, D., Jackson, E.A., \& Orr, D. (1995). Variation in sexual orientation among men who have sex with men, and their current sexual practices. Revue Canadienne de Santé Publique, 86, 384-388.

Parsons, J.T., Severino, J.P., Grov, C., Bimbi, D.S., \& Morgenstern, J. (2007). Internet use among gay and bisexual men with compulsive sexual behavior. Sexual Addiction \& Compulsivity, 14, 239-256.

Pink Triangle Services. (2001). How Well Are We Doing? A Survey of the GLBT Population of Ottawa. Retrieved from http://www.pinktriangle.org/wellness/report.pdf

Public Health Agency of Canada. (2010). National HIV prevalence and incidence estimates in Canada for 2008. HIV/ AIDS Epi Updates. Retrieved from http://www.phac-aspc.gc.ca/aids-sida/publication/epi/2010/pdf/EN_Chapter1_ Web.pdf

Ross, L.E., Dobinson, C., \& Eady, A. (2010). Perceived determinants of mental health for bisexual people: A qualitative examination. American Journal of Public Health, 100(3), 496-502.

Ryan, B., \& Chervin, M. (2000). Framing Gay Men's Health in a Population Health Discourse: A Discussion Paper. Ottawa, ON: Canadian HIV/AIDS Clearinghouse.

Saewyc, E.M., Homma, Y., Skay, C.L., Bearinger, L.H., Resnick, M.D., \& Reis, E. (2009). Protective factors in the lives of bisexual adolescents in North America. American Journal of Public Health, 99(1), 110-117.

Saewyc, E., Poon, C., Wang, N., Homma, Y., Smith, A., \& the McCreary Centre Society. (2007). Not Yet Equal: The Health of Lesbian, Gay, \& Bisexual Youth in BC. Vancouver, BC: McCreary Centre Society.

Smith, M. (2005, June 2-4). Explaining Human Rights Protections: Institutionalist Analysis in the Lesbian and Gay Rights Case. Paper presented at the Annual Meeting of the Canadian Political Science Association, University of Western Ontario, London, Ontario. Retrieved from http://www.cpsa-acsp.ca/papers-2005/Smith,\%20Miriam.pdf

Stall, R.D, Freeman, M., \& Catania, J.A. (2008). Interacting epidemics and gay men's health: A theory of syndemic production among urban gay men. In R.J. Wolitski, R.D. Stall, \& R.O. Valdiserri (Eds.), Unequal Opportunity: Health Disparities Affecting Gay and Bisexual Men in the United States (pp. 251-274). New York, NY: Oxford University Press.

StataCorp (2009). Stata Statistical Software: Release 11. College Station, TX: StataCorp LP.

Stokes, J.P., Miller, R.L., \& Mundhenk, R. (1998). Toward an understanding of behaviourally bisexual men: The influence of context and culture. The Canadian Journal of Human Sexuality, 7(2), 101-113.

Stokes, J.P., Vanable, P., \& McKirnan, D.J. (1997). Comparing gay and bisexual men on sexual behavior, condom use, and psychosocial variables related to HIV/AIDS. Archives of Sexual Behavior, 26, 383-397.

Tjepkema, M. (2008). Health care use among gay, lesbian, and bisexual Canadians. Health Reports, 19(1), 53-64.

Williams, T., Connolly, J., Pepler, D., \& Craig, W. (2003). Questioning and sexual minority adolescents: High school experiences of bullying, sexual harassment, and physical abuse. Canadian Journal of Community Mental Health, $22(2), 47-58$. 